J. theor. Biol. (1981) 89, 523-532

\title{
Sexual Competition and the Evolution of Hermaphroditism
}

\author{
Gerald Borgia $\dagger$ ANd James Blick \\ Museum of Zoology, University of Michigan, Ann Arbor, Michigan \\ 48109, U.S.A. \\ (Received 6 November 1978, and in revised form 10 August 1979) \\ The low density model produced by Tomlinson to describe the evolution of \\ hermaphroditism is shown to undervalue the level of gain to hermaph- \\ rodites in small populations. This model is revised to take into account gains \\ for hermaphrodites in breeding units where not only one but where both \\ sexes of gonochorists occur. This revision permits hermaphrodites \\ significant gains in populations as large as 50 individuals. Simulation results \\ are used to evaluate the revised model of hermaphroditism.
}

\section{Introduction}

Simultaneous hermaphroditism involving the production of both male and female gametes by the same individual at about the same time represents one of the few widespread alternatives to gonochoristic sexuality in living systems. Ghiselin (1974) discusses the distribution of this mode of reproduction among various phyletic groups including its occurrence in $95 \%$ of flowering plants.

Models for the evolution of this form of hermaphroditism have been reviewed in detail by Ghiselin $(1969,1974)$ and Charnov et al. (1976). In this paper we will present a reformulation of what Ghiselin has called the "low density" model. The model we develop shows a much greater advantage for hermaphrodites than earlier versions. This model is then related to others which have been used to explain the origin of hermaphrotidism. A comment is also made on the expected patterns of within-brood sex ratios in gonochoristic species based on the simulations presented in this paper.

\section{Reformulation of the Low Density Model}

Advocates of the low density model argue that in small breeding units gonochorists might all be of the same sex and therefore unable to find a

† Current address: Department of Biology, University of Chicago, Chicago, IL 60637, U.S.A.

(C) 1981 Academic Press Inc. (London) Ltd. 
suitable mate. Because hermaphrodites do not meet the same problem, they have an advantage when the likelihood of single-sex groups is high. Ghiselin (1969) attributes this form of the low density model to Darwin $(1851,1854)$ and Meyer (1888), but it was most clearly developed by Tomlinson (1966), who calculated the probability of unisexual groups in breeding units of different size. This model is attractive because of the common association of hermaphroditism in animals with low density situations (Fretter \& Graham, 1964; Ghiselin, 1969, 1974; Tomlinson, 1966) and because of unambiguous predictions it allows if specified density conditions are met.

Tomlinson's results show, however, that gain for hermaphrodites is rapidly reduced with even small increases in the size of breeding units. In groups of size eight or greater the calculated probability of unisexual groups occurring is less than one percent (see Fig. 1). Because advantage for hermaphrodites is dependent on the occurrence of the unisexual groups, there is a corresponding decrease in gain for hermaphrodites. Also important, but not considered by Tomlinson or Ghiselin, is the reduction of advantage to hermaphrodites as they increase in frequency in the population.

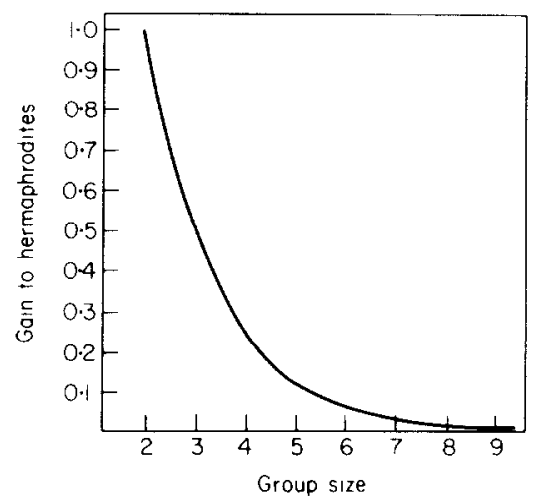

FIG. 1. Relative advantage to hermaphrodites over gonochorists due to the former's ability to avoid fertility losses in unisexual groups. This advantage is shown for groups of different size (after Tomlinson, 1966).

However, full advantage derived from hermaphroditism in small breeding units is not really considered in these earlier versions of the low density model. Local breeding units represent a small sample of an entire population and, as has been suggested, these units may vary greatly in composition by sex of gonochoristic members. Such variation in sexual composition allows an appreciable advantage for hermaphrodites over gonochoristic sexual 
competitors. This occurs because, on average, hermaphrodites produce a greater fraction of the gamates of the sex in short supply. Or, put another way, hermaphrodites produce a better sex-related investment ratio in structures and activities required for successful reproduction.

This effect can be understood if we consider the average gain for hermaphrodites and gonochorists in breeding units where males and females are present in unequal numbers. Following reasoning initially proposed by Fisher (1930), we may conclude that within any outbreeding unit individuals that invest in functions of the rarer sex should reproduce at a higher rate than those who produce those of more common form. Two conditions of sex ratio deviation are possible. First, if males are rare within a breeding unit, males may have a much higher expected reproductive output than females in the same group. As a result of the assumed sex ratio in these groups, most investment by gonochorists will be in female functions. If hermaphrodites are present their higher average investment in male functions allows them to enjoy genetic gain from the presence of abundant gonochoristic females. Here we assume that reproductive success in each sex is proportional to the level of investment by individuals in gametes of each type, and that individual hermaphrodites invest equally in the functions of each sex. If these assumptions hold, then hermaphrodites will out-reproduce gonochorists because individual hermaphrodites are, on average, able to invest more in the functions of the rarer sex. In the second case, when females are rare, the average male reproduces at a lower rate than the average female. Here hermaphrodites have a greater average investment in femaleness so they again produce more offspring, all else being equal, than gonochorists.

\section{Evaluating the Gains Due to Hermaphroditism}

The above description of the kinds of advantages hermaphrodites might enjoy in small breeding units, although accurate, gives no indication of the level of advantage we might expect. For this reason, we have carried out simulations which show the relative gain for hermaphrodites in groups of different size and differing proportions of hermaphrodites. In constructing our simulation models we assume a population of infinite size in which members of each sex are equally represented among the gonochoristic segment. Male, female and hermaphrodite offspring cost the same to make and in what might be equivalent to a free living larval stage are allowed to mix with others derived from different breeding units. Adult individuals randomly settle into closed breeding units. At breeding time all gametes are simultaneously expelled into the surrounding medium. Individual hermaphrodites produce one-half the number of eggs and sperm as 
gonochorists of the appropriate sex, and each male or hermaphrodite produces sufficient sperm, each sperm having equal probability of fertilizing the available eggs, to guarantee the fertilization of all the eggs produced by members of the breeding unit.

In this situation, three variables are considered: (1) size of breeding unit, (2) proportion of hermaphrodites in the source population, and (3) whether or not hermaphrodites fertilize their eggs (i.e. self). For each of the group sizes, reproductive outputs (total effective gametes) for gonochorists and hermaphrodites were calculated for all possible breeding unit combinations. These outputs are then weighted by the probability that each combination occurs in the population. After summing the weighted outputs, each total is divided by the population frequency of the respective type to give an average relative output per individual. The results are expressed as a ratio to give an index of the relative individual output for hermaphrodites compared to gonochorists. Separate gain ratios were calculated for the condition where sperm from a hermaphrodite were allowed to compete for that individual's own eggs, and in cases where they were not.

To illustrate the method employed to calculate the relative gain to hermaphrodites, consider the following example. Let the frequency of males $(M)$, females $(F)$, and hermaphrodites $(H)$ be $0 \cdot 2,0 \cdot 2$, and 0.6 respectively. Let the number of eggs produced by a female be $Y$ (so that a hermaphrodite produces $Y / 2$ ), and the number of sperm produced by a male be $X$ (so that a hermaphrodite produces $X / 2$ ). The probability of occurrence of each group is computed from the trinomial distribution as:

$$
P(I, J, K)=\frac{N ! \cdot M^{I} \cdot F^{J} \cdot H^{K}}{I ! \cdot J ! K !}
$$

where $I, J$, and $K$ refer to the number of males, females, and hermaphrodites respectively in a group of size $N$. Gametic output is calculated from the following:

(1) If the entire group is comprised of gonochorists of only one sex, then there are no effective gametes.

(2) If selfing can occur then the number of effective gonochoristic gametes (male and female) in a group is

$$
G_{g}=\frac{I \cdot X}{I \cdot X+K \cdot X / 2} \cdot(J \cdot Y+K \cdot Y / 2)+J \cdot Y .
$$

That is, $G_{\mathrm{g}}$ is equal to the gonochoristic proportion of the total sperm pool multiplied by the total number of eggs, plus the number of gonochorist female eggs. Similarly, the total number of effective hermaphrodite gametes 
is

$$
G_{h}=\frac{K \cdot X / 2}{I \cdot X+K \cdot X / 2} \cdot(J \cdot Y+K \cdot Y / 2)+K \cdot Y / 2
$$

(3) If selfing cannot occur then:

(a) if $K=1$ and $I=0$ then $G_{\mathrm{g}}=G_{h}=J$. $Y$;

(b) if $K>0$ then

$$
G_{g}=\frac{I . X}{I . X+K . X / 2} . J . Y+\frac{I . X}{I . X+(K-1) \cdot X / 2} \cdot K \cdot Y / 2+J . Y
$$

(note that when selfing cannot occur, a given hermaphrodite does not contribute sperm to fertilize its own eggs, hence the total number of sperm in competition for any set of hermaphrodite eggs is $I . X+(K-1) \cdot X / 2)$, and

$$
G_{h}=\frac{K \cdot X / 2}{I . X+K \cdot X / 2} \cdot J \cdot Y+\frac{(K-1) \cdot X / 2}{I \cdot X+(K-1) \cdot X / 2} \cdot K \cdot Y / 2+K . Y / 2
$$

\begin{tabular}{|c|c|c|c|c|c|c|c|c|}
\hline \multirow[b]{3}{*}{ Group } & \multirow{2}{*}{\multicolumn{3}{|c|}{ Composition }} & \multirow[b]{3}{*}{ Probability } & \multicolumn{4}{|c|}{ Total gametes } \\
\hline & & & & & \multicolumn{2}{|c|}{ Without selfing } & \multicolumn{2}{|c|}{ With selfing } \\
\hline & $M$ & $F$ & $H$ & & Gono & Herm & Gono & Herm \\
\hline 1 & 0 & 0 & 3 & 0.216 & 0 & $3 Y$ & 0 & $3 Y$ \\
\hline 2 & 0 & 1 & 2 & $0 \cdot 216$ & $Y$ & $3 Y$ & $Y$ & $3 Y$ \\
\hline 3 & 0 & 2 & 1 & 0.072 & $2 Y$ & $2 Y$ & $2 Y$ & $3 Y$ \\
\hline 4 & 0 & 3 & 0 & 0.008 & 0 & 0 & 0 & 0 \\
\hline 5 & 1 & 0 & 2 & 0.216 & $2 Y / 3$ & $4 Y / 3$ & $Y / 2$ & $3 Y / 2$ \\
\hline 6 & 1 & 1 & 1 & $0 \cdot 144$ & $13 Y / 6$ & $5 Y / 6$ & $2 Y$ & $\dot{Y}$ \\
\hline 7 & 1 & 2 & 0 & 0.024 & $4 Y$ & 0 & $4 Y$ & 0 \\
\hline 8 & 2 & 0 & 1 & 0.072 & $Y / 2$ & $Y / 2$ & $2 Y / 5$ & $3 Y / 5$ \\
\hline 9 & 2 & 1 & 0 & 0.024 & $2 Y$ & 0 & $2 Y$ & 0 \\
\hline \multirow[t]{2}{*}{10} & 3 & 0 & 0 & 0.008 & 0 & 0 & 0 & 0 \\
\hline & & & & 1.000 & & & & \\
\hline
\end{tabular}

For a group size of three, Table 1 gives the possible breeding groups with TABLE 1

their probabilities of occurrence and gametic outputs. The ratio of hermaphrodite to gonochorist output can be calculated as:

(A) WITHOUT SELFING

$$
\begin{aligned}
\text { Individual HERM }= & {[0 \cdot 216(3 Y+3 Y+4 Y / 3)} \\
& +0 \cdot 144((5 Y / 6)+0 \cdot 072(2 Y+Y / 2)] / 0 \cdot 6 \\
= & 3 \cdot 14 Y
\end{aligned}
$$




$$
\begin{aligned}
\text { Individual GONO }= & {[0 \cdot 216(Y+2 Y / 3)+0 \cdot 144(13 Y / 6)} \\
& +0 \cdot 072(2 Y+Y / 2)+0 \cdot 024(4 Y+2 Y)] / 0 \cdot 4 \\
= & 2 \cdot 49 Y
\end{aligned}
$$

Ratio HERM/GONO $=3 \cdot 14 Y / 2 \cdot 49 Y=1 \cdot 2610$

(B) WITH SELFING

$$
\begin{aligned}
\text { Individual HERM }= & {[0 \cdot 216(3 Y+3 Y+3 Y / 2)+0 \cdot 144(Y)} \\
& +0 \cdot 072(3 Y+3 Y / 5)] / 0 \cdot 6 \\
= & 3 \cdot 372 Y
\end{aligned}
$$

Individual GONO $=[0 \cdot 216(Y+Y / 2)+() \cdot 144(2 Y)+0 \cdot 072(2 Y+2 Y / 5)$

$$
\begin{aligned}
& +0 \cdot 024(4 Y+2 Y)] / 0 \cdot 4 \\
= & 2 \cdot 332 Y
\end{aligned}
$$

Ratio HERM $/$ GONO $=3 \cdot 372 Y / 2 \cdot 322 Y=1 \cdot 4522$

These kinds of calculations were carried out for various group sizes and varying population frequencies of hermaphrodites. The results (see Table 2) show relatively high gain ratios for hermaphrodites even in groups as large as 50 individuals, either with or without inbreeding. This advantage is maintained for conditions where hermaphrodites are present even in high frequency. These results contrast sharply with those of earlier versions of the low density model which allow gain to hermaphrodites only when they are associated with unisexual groups of breeding populations.

\section{Discussion}

The low density model as conceived here deals expressly with conditions where the size of the breeding unit is limited and is composed of individuals with low levels of genetic relatedness, as is likely when dispersal occurs before sexual maturity. The small size of breeding unit may occur because of physical containment in a patchily distributed medium, such as a tide pool, where successful dispersal of gametes between local aggregations of conspecifics is unlikely. Also, fixed positioning of sexually mature individuals, as in plants, may limit the number of effective mates in a given area. Thus even when individuals are not contained in physically distinct units, there may be a limited range over which an individual's gametes are likely to be effective, thereby defining an effective breeding unit for each individual 


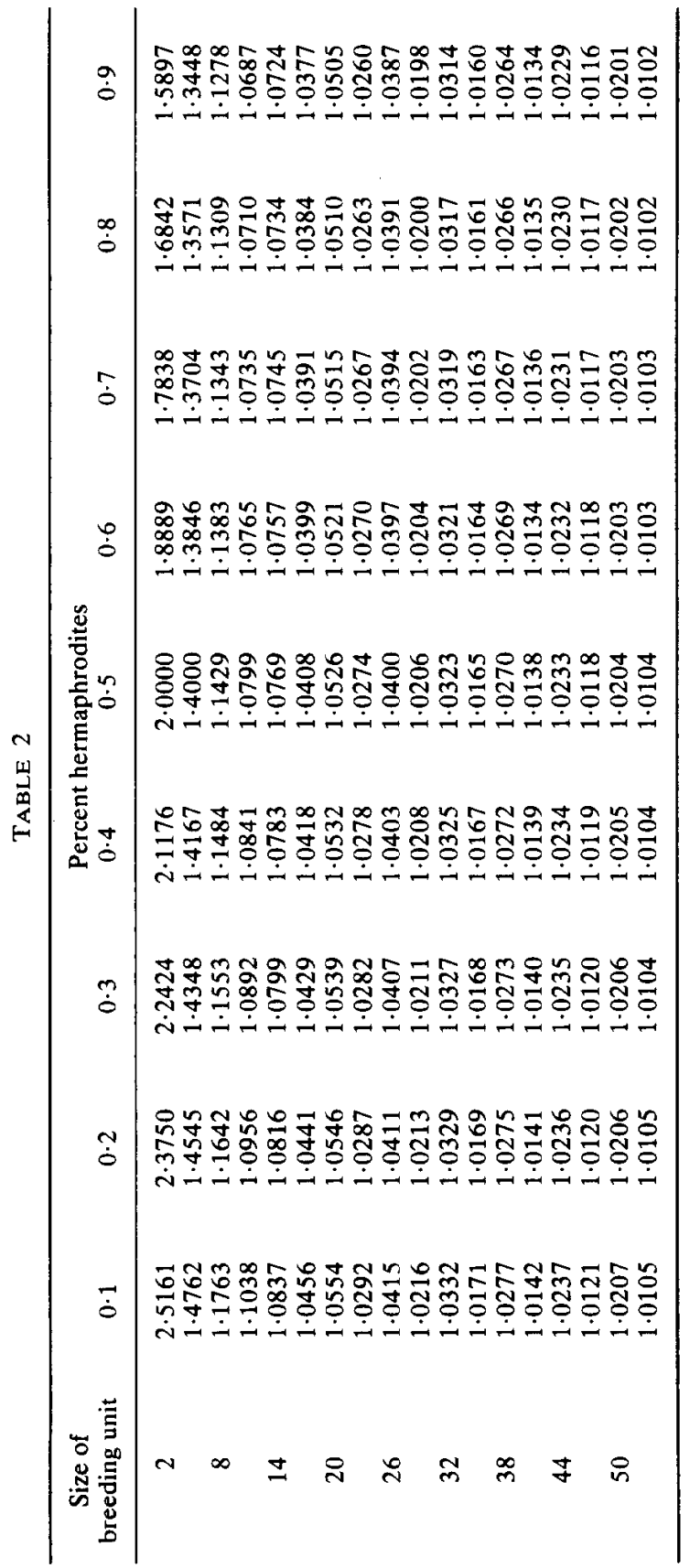


which may largely overlap with those of nearby conspecifics. In plants such effects may be enhanced in cases where pollinators forage over limited areas.

Application of the model need not be restricted to conditions of more or less random shedding of gametes into the external medium. Pairing of individuals for fertilization and even internal fertilization might provide suitable conditions for its effects to be important. However, this is unlikely because of expected tendencies for hermaphrodites to be less effective at overt sexual competition and for mating preferences to be tied to differences in reproductive gain associated with selection of hermaphrodites and gonochorists as mates.

Two other models, the low mobility model (Charnov et al., 1976) and the sampling error model (Murray, 1964; see also Ghiselin, 1969) may be complementary and also depend upon small effective breeding units to have important effects on the success of hermaphrodites. Even though in all of these models gains for hermaphrodites are inversely related to the size of the breeding unit, unlike the low density model, the magnitude of effects for each of these models cannot be estimated without detailed information from natural populations. Two other models discussed by Charnov et al. (1976), those of cost sharing and resource overlap, are even more difficult to evaluate because their effects are not directly dependent on group size or any other easily defined population parameter.

The low density model considers gains for hermaphrodites with fixed investment patterns. In cases where individuals can perceive the sex ratio of their breeding unit, hermaphrodites could achieve a reproductive advantage by varying investment patterns so that gametes in short supply are preferentially produced. Obviously hermaphrodites are in a better position than gonochorists to make adjustments of this type since they can become either sex. Investment patterns in sex products for gonochorists apparently can only be adjusted at the time eggs are fertilized (see Hamilton, 1967). Thus the parent must anticipate investment ratios for its gonochoristic offspring and, as pointed out by Borgia (1980) there are reasons to expect that such biases will be rare except in cases of post-fertilization investment in offspring. This suggests another situation in which hermaphrodites might out-reproduce gonochorists. The degree of adjustment might be complete, as in the case of sequential hermaphrodites, where the expected success of members of each sex can be predicted; or partial as in cases where there is a significant probability that one or the other sex is in short supply but there is still a high level of uncertainty.

Our model showing gains for hermaphrodites at low density is similar to the one proposed by Verner (1965) in which he suggests that parents who tend to produce equal within-brood sex-related investment in offspring 
should be favored by selection over those producing a more variable investment pattern. Parents producing sons and daughters in equal numbers have more grandchildren than those producing more variable sex ratios because their offspring are more successful in sexual competition. Like the gain for hermaphrodites in the low density model, the level of gain for gonochorists resulting from equal sex-related investment in the next generation is dependent on the size of groups and the deviations from equal sex ratio in those groups.

As we have shown, balanced investment by hermaphrodites in gametes leads to immediate advantage in sexual competition over parents who vary their investment in sex-related activities. However, the effect Verner describes for gonochorists occurs only after offspring have reached sexual maturity and compete for mates in the same small breeding unit. The time delay between when gonochoristic parents produce investment biases and when offspring mature increases the likelihood of offspring dispersal from their natal group. Such dispersal seems common in most species as a result of individual attempts to avoid inbreeding. Hence, even in populations of very similar structure, hermaphrodites may gain a very large advantage from an even investment pattern in gametes while gonochorists show little real gain from balancing the sex of offspring in their broods.

Evolution of the ability to control the sex of offspring within broods may be complicated in other ways. In small populations which consistently show little or no dispersal the lack of genetic variation arising from endogamous matings reduces much of the gain from sexual reproduction. Close inbreeding might be important in these populations because it allows for shifts in investment favoring daughters with no loss in the reproductive gains for parents through sons (Hamilton, 1967). Female-biased sex ratios which Hamilton suggests are the expected result from inbreeding would tend to extinguish selection for equal within-brood investment. Thus small endogamous populations which are most likely to meet the conditions necessary for the evolution of equal within-brood sex ratios are also those in which sex ratio adjustment due to inbreeding is likely to occur.

The evolution of investment biases in offspring of each sex is further complicated by the apparent inability of most gonochoristic species to deterministically control the sex of offspring they produce. In almost all diplo-diploid species sex determination appears to be random. It has been suggested that such a system may have evolved in response to frequent meiotic drive of sex determining chromosomes (see Alexander \& Borgia, 1978; Borgia, 1980). The vast majority of gonochoristic species that do show a high degree of control over the sex of their offspring are haplo-diploid; a sex determining system which is immune to the effects of 
metiotic drive of sex chromosomes and the resulting population-wide biases in sex ratio.

Thus, in addition to the effects of dispersal in removing much of the selective pressure favoring equal investment within broods, it is unlikely that diplo-diploid sex determining systems which differ from those involving random sex determination often evolve. Under conditions that are suitable for the evolution of sex ratio equalization within broods, and even where control of sex ratio is possible, selection for sex ratio equalization most likely will be overcome by an even stronger tendency for inbreeding and the evolution of sex ratio biases favoring females. So even though the kind of selection pressures Verner describes may operate in some cases, they are likely to be weak, often nullified by opposing effects.

\section{REFERENCES}

Alexander, R. D. \& Borgia, G. (1978), Ann. Rev. Ecol. Syst. 9, 449.

Borgia, G. (1980). Theor. pop. Biol. (in press).

Charnov, E.. Maynard Smith, J. \& Bull, J. 119761. Nature 263, 125.

DARWIN, C. (1851). A Monograph on the Sub-Class Cirripedia, with Figures of All the Species. The Lepadidae; or Pedunculated Cirripedes. London: Ray Society.

DARWIN, C. (1854). A Monograph on the Sub-class Cirripedia, with Figures of All the Species. The balanidae (or Sessile Cirripedes); the Verrucidae, etc. London: Ray Society.

FISHER, R. (1930). The Genetical Theory of Natural Selection. New York: Dover.

Fretter, V.\& Graham, A. (1964). In Physiology of the Mollusca. (K. Wilbur \& C. Yonge. edsi New York, London: Academic Press.

GHiselin, M. (1969). Q. Rev. Biol. 44, 189.

GHiSELIN, M. (1974). The Economy of Nature and the Evolution of Sex. Univ. of California Press.

Hamilton, W. D. (1967). Science 156, 477.

MeYer. E. (1888). Mitt. Zool. Stat. Neapel. 8, 462.

Murray, J. (1964). Evolution 18, 283.

TOMLINSON, J. (1969). J. theor. Biol. 11, 54.

VERNER, J. (1965). Am. Nat. 99, 419. 